\title{
Clinical outcome when left atrial posterior wall box isolation is included as a catheter ablation strategy in patients with persistent atrial fibrillation
}

Submitted: 28 January 2016; Accepted: 01 March 2016; Published online: 04 March 2016

Despite on-going advances in the field of catheter ablation, the optimum strategy for ablation of persistent atrial fibrillation (AF) remains unclear. While pulmonary vein isolation (PVI) remains the cornerstone of treatment for $\mathrm{AF}$, successes rates remain considerably lower in patients with persistent versus paroxysmal AF.

The technique of posterior left atrial wall box isolation has been in increasing use since first described by Kumagai in 2007 [1] however only limited and conflicting data exists with regards to its efficacy. The rationale behind isolation of left atrial wall is based on its common embryonic origin with that of the pulmonary veins and the frequent finding of drivers and rotors in this area. In our small single centre study [2], we sought to evaluate a strategy of PVI plus posterior left atrial box isolation, consisting of a left atrial (LA) roof line and inferior transverse line in a group of 100 patients whose pattern of AF was predominantly persistent $(72 \%)$. Isolation of the posterior left atrial wall was achieved in all but one of our patients. Freedom from recurrent AF on follow-up was achieved in 75 $\%$. Obvious limitations include the size of the study and lack of a comparative arm. More specifically the majority of our patients who remained arrhythmia free also underwent additional ablation to sites of high frequency activity elsewhere in the atria based on intraprocedural findings and clinical factors at the discretion of the operator. While this limited our ability to attribute success solely to the box isolation set it did suggest that our 'real world' strategy of box isolation combined with more extensive substrate ablation as deemed appropriate may be an effective one particularly when compared to other real world data of PVI alone for patients with persistent AF. In addition there were no adverse outcomes associated with this more extensive procedure. Our findings support the current guidelines that recommend adjuvant substrate modification in addition to pulmonary vein isolation in persistent $\mathrm{AF}$. The STAR AF 2 trial, which was presented at the ESC by Dr. Verma in 2014 and the results of which were published in the New England Journal of Medicine earlier this year, casts doubt over these guidelines [3]. STAR AF 2 is significant in that it is one of the few recent large scale randomized multi-centre trials comparing ablation strategies in persistent $\mathrm{AF}$ (Table 1). Patients were randomly assigned to PVI alone, PVI plus ablation of complex fractionated atrial electro grams (CFAEs) or PVI plus linear ablation of LA roof line and mitral isthmus line. At

18 months rates of the primary outcome of freedom from atrial fibrillation after one
Louisa O’Neill* \& David Keane Department of Cardiology, St Vincent's University Hospital, Dublin, Ireland *Author for correspondence: Tel.: 00353879136176 louisaoneill@yahoo.co.uk 


\begin{tabular}{|c|c|c|}
\hline & STAR AF 2 Trial Verma et al. & $\begin{array}{l}\text { 'Clinical outcome when left } \\
\text { atrial box isolation is included } \\
\text { as a catheter ablation strategy } \\
\text { in patients with persistent atrial } \\
\text { fibrillation' O'Neill et al. }\end{array}$ \\
\hline Type of study & Multicentre, randomised controlled trial & Single centre, non-comparative series \\
\hline Patient numbers & 589 & 100 \\
\hline Pattern of AF & Persistent - $100 \%$ & Persistent-72\% Paroxysmal-28\% \\
\hline Lesion set & $\begin{array}{l}\text { PVI vs PVI plus mitral isthmus and LA roof line vs PVI plus ablation of } \\
\text { CFAEs }\end{array}$ & PVI plus LA box isolation \\
\hline $\begin{array}{l}\text { Follow up } \\
\text { monitoring }\end{array}$ & $\begin{array}{c}12 \text { lead ECG and Holter monitoring at 3,6,9,12 and } 18 \text { months. } \\
\text { Trans telephonic monitor for duration of follow up }\end{array}$ & $\begin{array}{l}\text { Holter monitoring at 2, } 6 \text { and } 12 \\
\text { months }\end{array}$ \\
\hline
\end{tabular}

procedure were not significantly different between time was longer for those receiving supplemental groups. Lower success rates were seen when compared ablation although actual rates of adverse events were to our study. In addition, procedure and fluoroscopy similar between groups (Figure 1).

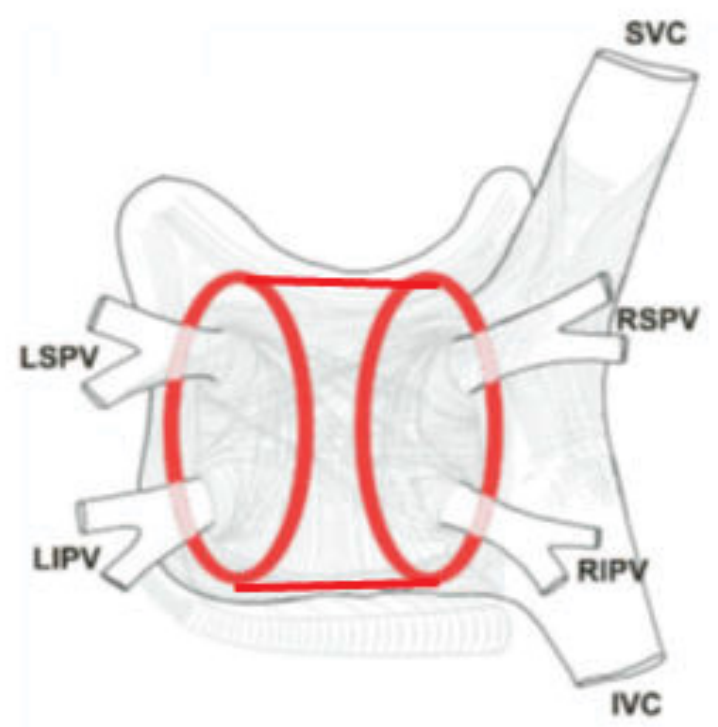

Figure 1: Posterior view of left atrium illustrating lesion set for PVI and posterior LA box isolation.

\section{References}

1. Kumagai K, Muraoka S, Mitsutake C, Takashima H, Nakashima H. A new approach for complete isolation of the posterior left atrium including pulmonary veins for atrial fibrillation. J. Cardiovasc. Electrophysiol. 18, 1047-1052 (2007).

2. O'Neill L, Hensey M, Nolan W, Keane D. Clinical outcome when left atrial posterior wall box isolation is included as a catheter ablation strategy in patients with persistent atrial fibrillation. J. Interv. Card. Electrophysiol. 44, 63-70 (2015).

3. Verma A, Jiang CY, Betts TR, et al. Approaches to Catheter Ablation for Persistent Atrial Fibrillation. N. Engl. J. Med. 372, 1812-1822 (2015). 